\title{
Lobular carcinoma of the breast: a special variant of mucin-secreting carcinoma
}

\author{
A. GAD AND J. G. AZZOPARDI
}

From the Department of Histopathology, Royal Postgraduate Medical School, London

SYNOPSIS There is currently much speculation over the precise nature of lobular carcinoma in-situ of the breast and its significance. Histochemical study shows that it is rich in sialomucin. This has a characteristic intracellular distribution which distinguishes lobular carcinoma in-situ from cancerization of lobules. The same histochemical features are seen in the infiltrative phase of the tumour. These findings invalidate the concept that it is a myoepithelial-cell tumour. Practical applications of the distinctive pattern of mucin secretion are discussed.

Foote and Stewart (1941) first clearly defined the entity of lobular carcinoma in-situ of the breast. This entity has achieved widespread recognition, especially over the last decade. Warner (1969) gave an excellent account of the disease and the associated problems, to which little of substance has been added since. Most of the emphasis in recent years has been on the prognostic and therapeutic implications of the in-situ variety and on its mammographic recognition. The infiltrative form of lobular carcinoma also has a distinctive microscopic appearance (McDivitt et al, 1968). One of the many curious aspects of lobular carcinoma is the distinction between it and so-called cancerization of lobules (Fechner, 1971), a distinction which is maintained in the infiltrative phase of the respective tumours. Hamperl (1972) has suggested that it is of myoepithelial cell derivation.

Impressed by the generally accepted observation that it originates in lobules and yet forms no ductal structures as seen with 'scirrhous carcinoma' (infiltrating duct carcinoma), it occurred to us that lobular carcinoma might represent a variant of a secretory type of tumour. To our surprise we found no mention of mucin study in the now extensive literature on lobular carcinoma of the breast.

\section{Materials and Methods}

Twenty cases of lobular carcinoma of the breast were selected for study. These were taken from the files of the Department of Histopathology at the Royal Postgraduate Medical School. The 20

Received for publication 12 March 1975. tumours were from 19 different patients since, though four of these patients had bilateral primary tumours, the second tumour was removed in three cases outside the period of study. Of the 20 cases, 10 had lobular carcinoma in-situ only, three had in-situ and infiltrating lobular carcinoma, and seven had infiltrating lobular carcinoma in the blocks studied. The material included both excision biopsy and mastectomy specimens. Patients in whom only lobular carcinoma in-situ was found were all operated on for what was clinically regarded as benign breast disease. The benign lesions found in these patients are not considered further here.

For purposes of comparison with lobular carcinoma in-situ, six cases of cancerization of lobules as defined by Fechner (1971) were studied simultaneously by the same methods.

Tissues were fixed in $10 \%$ neutral buffered formalin and processed in routine fashion, and paraffin sections were cut at $5 \mu$. In addition to haemalum eosin staining, a series of histochemical stains for mucosubstances as used by Gad (1969) was employed on all cases. This includes: periodic acid Schiff (PAS) with and without diastase pretreatment, alcian blue-periodic acid-Schiff sequence (AB/PAS) with and without nuclear counterstain with haemalum, alcian blue with neutral red nuclear counterstain $(\mathrm{AB} / \mathrm{NR})$, and high iron diamine alcian blue combination (HID/AB). Digestion experiments were carried out as follows:

SIALIDASE AB/PAS Air-dried sections were incubated in sialidase (neuraminidase, purified Vibrio cholerae) at a strength of 100 units per $\mathrm{ml}$ in $0.5 \mathrm{M}$ acetate buffer containing $0 \cdot 10 \%$ calcium chloride at $\mathrm{pH} 5.5$ 
for 24 hours at $37^{\circ} \mathrm{C}$. Removal of sialic acid is shown by loss of alcianophilia.

HYALURONIDASE AB/PAS Air-dried sections were incubated in $0.05 \%$ solution of bovine testicular hyaluronidase (Type I, 412 NF units per gram) at $\mathrm{pH} 5.5$ for 16 hours at $37^{\circ} \mathrm{C}$.

\section{Results}

The cells of both in-situ and infiltrating lobular carcinoma contain rounded intracytoplasmic globules of mucosubstance (figs 1 to 3 ). The percentage of positive cells varies in different areas between as little as $7 \%$ and as much as $75 \%$ with an average of about $25 \%$. The mucosubstance is alcianophilic and is PAS positive after diastase digestion. Cells contain one to several (up to 6) globules in a $5 \mu$ section. The globule or globules may be minute and difficult to see, small but easily visible, or large enough to occupy half of the cell. The single globules may be small or large (fig 4), the multiple globules forming a conglomerate of small vacuoles. Excellent fixation is essential for the proper demonstration of the secretory product. In material in which fixation has been delayed, tumour cells may show more or less diffuse staining, and the vacuolar character of the mucin may not be very evident.

With alcian blue, the globules of mucosubstance do not stain uniformly: in most instances there is a peripheral blue rim with a sharp outer margin and an often less distinct inner edge. This blue rim may be extremely fine and delicate or quite broad: similarly, it varies in staining intensity from a light to a dark blue (fig 3). The core of the globule usually stains faintly or not at all with alcian blue. Somecells contain a dot or globule of mucin situated within the faintly staining or unstained core. Like the outer halo, this may stain a pale or dark blue. This 'target' appearance is very characteristic of the staining reactions of the mucin in this tumour (fig 3). It is not clear what the unstained or 'empty' space represents. While it may, in part at least, represent a retraction artefact, alternative possibilities must be considered. Ozzello (1971) demonstrated that tumour cells of lobular carcinoma contain intracellular lumina lined by microvilli. Personal studies (Eusebi and Azzopardi, unpublished data) have confirmed this observation. It is possible that the mucin halo represents mucin entangled in intracellular microvilli while the central mucinous dot represents mucin free in the intracellular lumen. Ultrastructural mucin staining should settle whether or not this interpretation is correct.

With PAS, after diastase, there is variable depth of staining of both the peripheral rim and the central $\stackrel{0}{\vec{*}}$

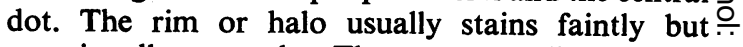
occasionally strongly. The same applies to the $\overrightarrow{\vec{F}}$ central dot, though this stains deeply more often than the halo. Thus, in the AB/PAS combination the central dot may stain red or purple rather than $\frac{\bar{\sigma}}{\overline{\frac{D}{}}}$ blue, producing a very striking two-tone target $\frac{\rho}{\Phi}$ zone (fig 5). This appearance is seen most commonly $\cong$ in the in-situ carcinomas. In a few cells the secretion कै stains a grey to brown colour with the HID/AB $\vec{\circ}$ combination. Tumour cells of infiltrating lobular carcinoma have essentially similar staining reactions $\vec{\omega}$ (figs 6 and 7).

These staining reactions indicate that a non- $\frac{?}{0}$ sulphated acidic mucosubstance is the major secretory product of lobular carcinoma of the breast. 6 This dominant component is sialidase-susceptible $\searrow$ and resistant to hyaluronidase digestion, indicating $\vec{O}$ that it is sialomucin. The HID/AB combination $\frac{\text { ? }}{2}$ shows that, in addition to sialomucin as the $\vec{c}$ dominant component, sulphomucin is occasionally $\mathbb{D}$ present in a few tumour cells. Neutral mucosubstance is also present, espeically in the centre of the globules, when a red or purple dot is seen with $\mathbb{\Phi}$ PAS after prior staining with AB.

Nuclear counterstaining with haemalum of जै neutral red shows beyond doubt that the muco substance secreted is exclusively intracellular. nuclear counterstain is not, however, recommende for general use as it has some masking effect on the mucin staining, even for those who are familiar

Fig 1 Lobular carcinoma in-situ affecting two adjacent lobules. Alcian blue PAS $\times 160$

Fig 2 Numerous mucinous vacuoles in neoplastic cells of LISC. Alcian blue PAS $\times 480$

Fig 3 Higher power view of part of fig 2. Note mucinous haloes with unstained core usually containing a mucinous globule. There is marked variation in the staining properties of different vacuoles. AB/PAS $\times 720$

Fig 4 LISC with intracellular mucin. Note marked variation in size of haloes. $A B / P A S \times 840$

Fig 5 Central globule of red-staining neutral mucosaccharide is separated by clear space from alcianophilic haloes. The stromal cells at top and bottom are mast cells. $A B / P A S \times 840$

Fig 6 Infiltrating lobular carcinoma with innumerable rounded alcianophilic vacuoles. $A B / P A S \times 160$

Fig 7 Alcianophilic haloes in tumour cells of infiltrating lobular carcinoma. $A B / P A S \times 480$

Fig 8 Same case as in fig 7. Note the masking effect of a nuclear counterstain on the identification of the intracellular mucin. AB/PAS and haemalum $\times 640$ 


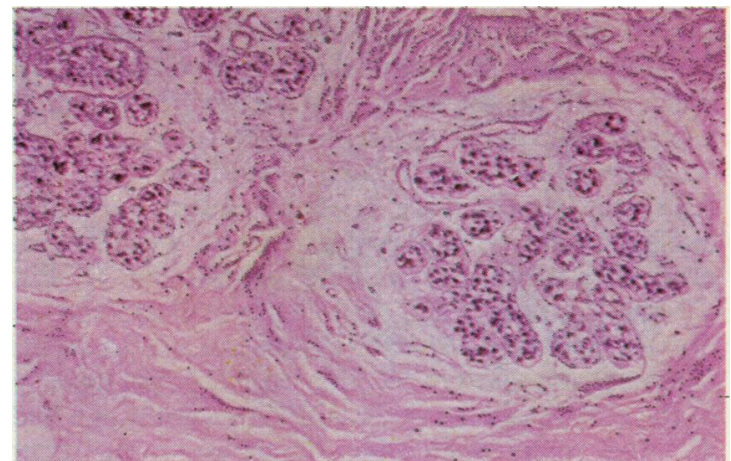

Fig. 1

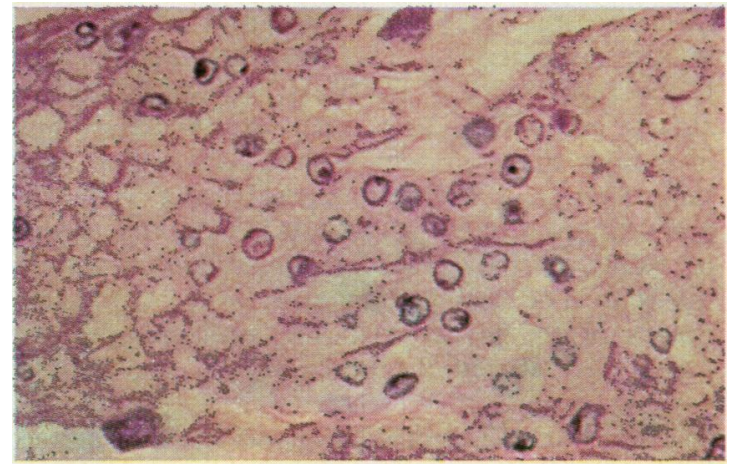

Fig. 3

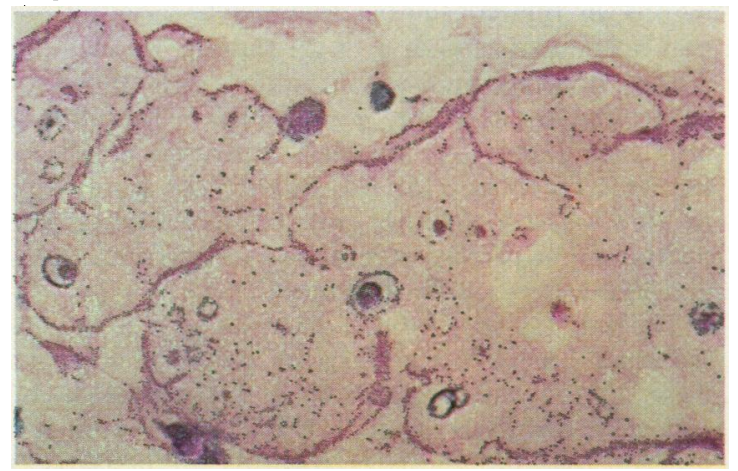

Fig. 5

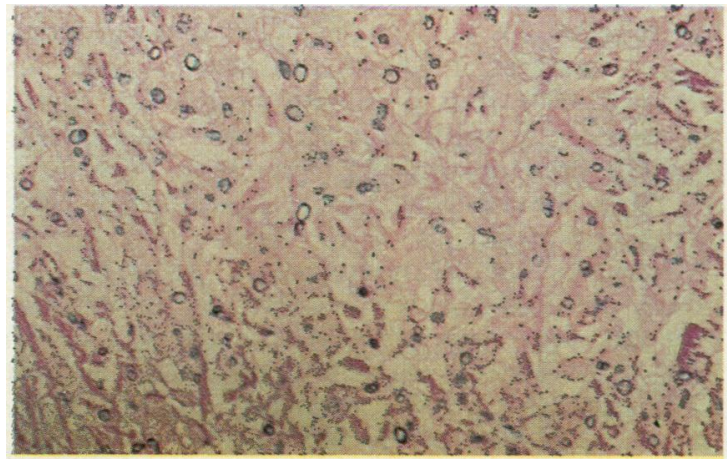

Fig. 7

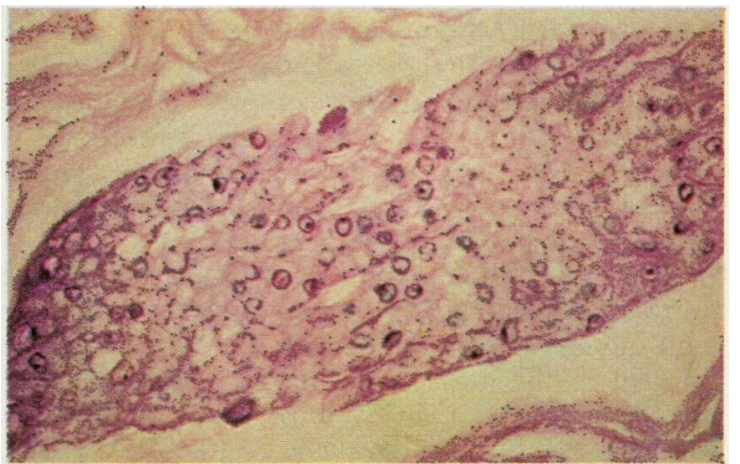

Fig. 2

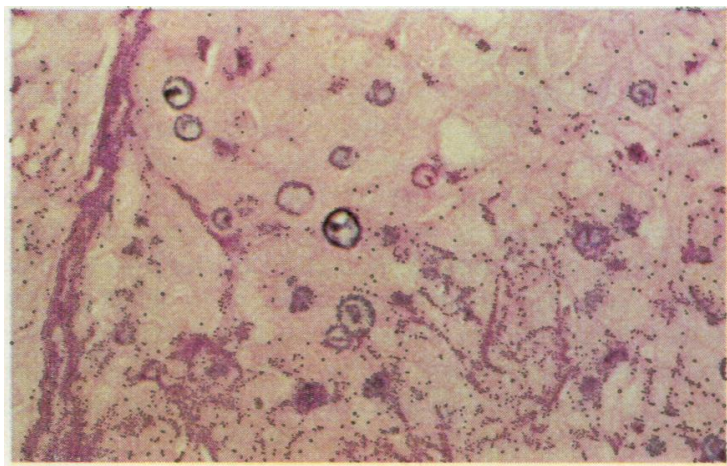

Fig. 4

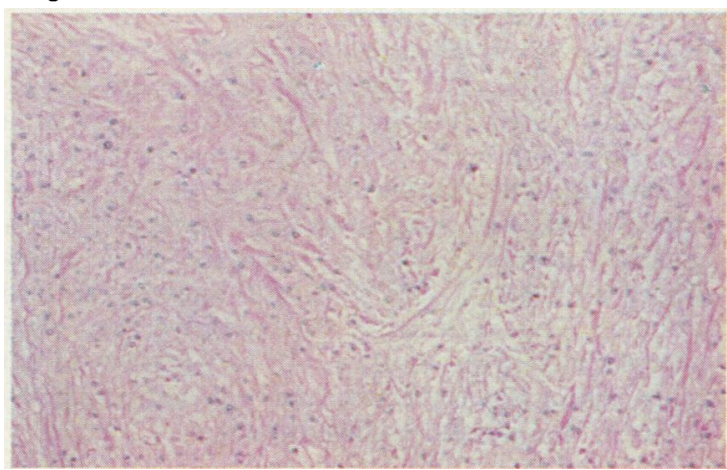

Fig. 6

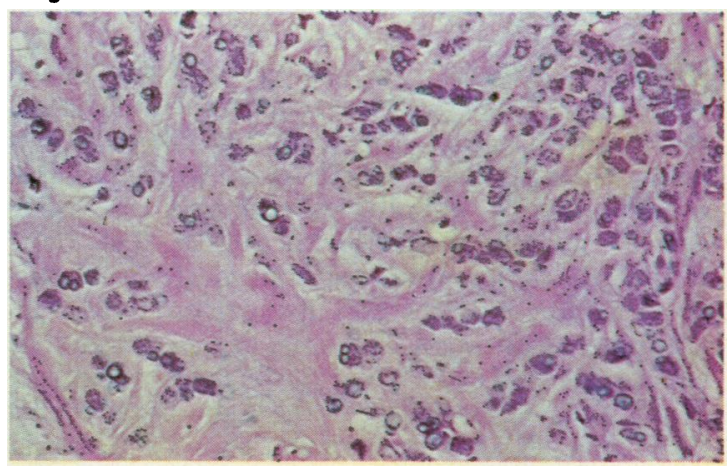

Fig. 8 
with the AB/PAS combination (fig 8). Though the nucleus may be slightly eccentric, the intracellular globules of secretion do not compress it against the cell membrane so that a signet-ring appearance is not produced. In some cells, the nucleus is overlapped by the secretory globule without any appearance of indentation or compression.

Numerous mast cells are often present in the intralobular connective tissue in both neoplastic and non-neoplastic areas (fig 5). They are also present in the interlobular stroma. The mast cells are filled with prominent, dark brown granules in the HID/AB preparations. The position of the cells, their intense granularity, and the depth of staining with HID should prevent any confusion with tumour cells. The depth of staining of tumour cells with HID very rarely even approaches that seen in mast cells, signifying, as already indicated, only a minor component of sulphomucin in tumour cells.

The AB/PAS and the HID/AB combinations map out clearly foci of neoplastic cells, whether within the units of a lobule, the ductule or the interlobular duct. Affected areas stand out in stark relief against the background of normal tissues. AB/PAS without nuclear counterstain is the most satisfactory for general purposes as the architectural outlines are more clearly seen than with HID/AB.

\section{CANCERIZATION OF LOBULES}

The secretion in cancerization of lobules is almost all intraluminal. The spaces of varying size among the tumour cells are lined by a fuzzy layer of a mucosubstance with the staining characteristics of a sialomucin. The cytoplasm of most of the tumour cells is devoid of mucosubstance. However, a few cells do contain variable amounts of a finely granular material which is not aggregated into globules. This material is PAS positive even after pretreatment with diastase. It is negative to all other techniques for acidic material, indicating the presence of a neutral mucosubstance.

\section{Discussion}

Lobular carcinoma, both in-situ and infiltrating, is a tumour that secretes acidic mucosubstances: these are intracellular in location. The mucosubstance consists mainly of sialomucin with a variable, usually minute, quantity of sulphomucin. Some neutral mucosubstance is also present. The alcian blue PAS combination, without nuclear counterstain, is the best method for demonstrating the presence, localization and quantity of these components. Alcian blue at $\mathrm{pH} \mathbf{2 . 5}$ demonstrates sialomucin and sulphomucin in the tumour cells while PAS, after alcian blue staining, shows up residual neutral mucosubstances, unstained by alcian blue. Sialidase digestion confirmed that the dominant acidic $\bar{\sigma}$

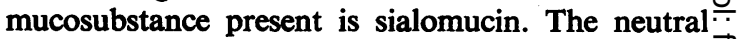
mucopolysaccharide present tends to occupy the $\overrightarrow{\vec{F}}$ central position in the vacuole so that in the $\mathrm{AB} / \mathrm{O}^{-}$ PAS preparation there is sometimes a large blue $\frac{}{0}$ circle or halo with a central red (or purple) dot, $\overline{\overline{\bar{p}}}$

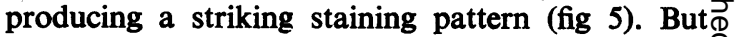
a 'target' appearance, with a central globule or dot separated by a space from an outer halo, is the $\mathrm{C}^{\mathrm{N}}$ characteristic pattern of this tumour, irrespective $\vec{O}$ of the colour scheme in the AB/PAS stain (figs 3 and $\overrightarrow{-}$ 4). The main qualitative difference from other ${ }_{\sigma}^{\omega}$ carcinomas of the breast is the secretion by lobularo carcinoma of a very small amount of sulphomucin, as demonstrated by the HID/AB combination. $\infty$ Mucoid carcinoma of the breast secretes sialomucin almost exclusively, and other breast carcinomas, $=$ including the common 'scirrhous', secrete both $\vec{\circ}$ sialomucin and neutral mucosubstance (Gad, in preparation). But it must be stressed that the amount $\vec{c}$ of sulphomucin in lobular carcinomas is usually $\mathbb{D}$ minimal. The major difference from other carci- $\mathbb{\Phi}$ nomas of the breast is in the localization of the $\bar{\sigma}$ mucin-exclusively intracellular in the lobular $\frac{\mathbb{\Phi}}{-}$ variety, dominantly extracellular or in tubular $\overrightarrow{0}$ structures in the mucoid and scirrhous varieties. जr While in scirrhous carcinoma the mucin is dominantly in tubular lumina, it must be pointed ofito that occasional tumour cells do contain intracellular mucin droplets indistinguishable from those of infiltrating lobular carcinoma. The proportion of cells containing such droplets is usually small, of 2 the order of only 2 or $3 \%$ but it may reach $5 \% . \overrightarrow{0}$ In view of this, it is not possible to exclude some 3 overlap in the pattern of mucin secretion of scirrhous and infiltrating lobular carcinoma.

The finding that lobular carcinoma is a mucin- $\overline{-}$ secreting type of tumour is at variance with the view that it is exclusively a myoepithelial-cell 3 . cancer or dysplasia (Hamperl, 1972). Clearly it is a tumour that differentiates towards epithelial cells $̧$ and dominantly towards secretory cells. It is $\mathrm{O}$ conceivable that lobular carcinoma shows differen- tiation towards myoepithelial cells as well aso epithelial cells. This possibility must be considered in view of the work of authors who found 'large $N$ numbers of cytoplasmic fibrils similar in appearancen to those seen in recognizable myoepithelial cells' $N$ at the ultrastructural level (Carter et al, 1969). We are concerned at the frequency with which such $\%$ fibrils are found in a whole variety of different tumours, for example, Wellings and Roberts (1963)ळ found very similar appearances in infiltrating duct ${ }^{+}$ carcinoma. For present purposes, suffice it to say that identification of myofibrils in many tumours, $\overrightarrow{\mathbb{D}}$ 
including breast carcinomas, is fraught with difficulties, and that we have reservations about the type of evidence presented to date. Certainly the evidence that lobular carcinoma shows epithelial differentiation is incontrovertible.

The tumour cells secrete intracellular mucin globules and are 'dissociated' one from another, this being seen most clearly in the infiltrative phase. This is analogous with tumours in other organs (for example, stomach), in which tumours with dominantly intracellular mucin secretion show lack of cohesiveness, expressed as cell 'dissociation', associated with markedly infiltrative activity.

Staining for mucin with the AB/PAS combination is useful as a method of scanning breast tissue at low magnification both for the detection of lobular carcinoma in-situ as well as for mapping out the extent of lobular involvement and ductal Pagetoid spread. Lobular carcinoma in-situ is easily missed and there is reason to believe that it is not diagnosed as frequently as it should be. Mucin stains are of great value to those who are not very conversant with this disease: to the expert, admittedly, this method is only slightly better than scanning haemalum-eosin sections.

The constancy of the pattern of mucin secretion makes the distinction from other types of breast carcinoma easier and more objective. This helps in a number of practical ways. First, it adds a new criterion to the distinction between lobular carcinoma in-situ (LISC) and lobular cancerization, since in the latter most of the mucin is luminal. Secondly, Newman (1966) distinguished 'pure' and mixed forms of lobular carcinoma as he recognized that the distinction between lobular and ductal carcinoma was not always as clear-cut as in the classical forms of the diseases. In this situation an objective criterion, like that described here, should help in the differentiation. Thirdly, this method can help reduce some of the confusion that has crept into the literature. Some authors have not attempted to distinguish between ductal carcinoma and lobular carcinoma on the grounds that both are composed sometimes of what is called a 'small cell' (Toker, 1974). Figures 10 and 11 of this author unfortunately do not represent the lesion described over the last three decades by the Memorial Hospital group of workers and many others. This lumping together of different entities, on the sole and dubious criterion of cell size, will confuse the essential quality of LISC, as ably summarized by Warner (1969), apart from rendering impossible valid conclusions about the prognostic significance of the separate entities. Application of mucin stains in future will help prevent the unwary from falling into similar traps.
It is well known that metastases in nodes from infiltrating lobular carcinoma can mimic malignant lymphoma (McDivitt et al, 1968). Alcian blue/PAS staining resolves this objectively and categorically. With metastases in sites like the skin, this method is also useful, both in diagnosing metastatic carcinoma and even in suggesting the primary site of origin where this has not yet made itself manifest.

It is a very curious fact that the intracellular mucin in tumour cells of lobular carcinoma rarely seems to aggregate or stain in such a way as to give rise to signet-ring cells. Why this is so is not clear but, were it otherwise, the nature of the tumour would have become obvious a long time ago. The question that springs to mind is whether the rare signet-ring cell carcinoma of the breast is not, in some cases at any rate, the extreme and easily recognizable expression of an infiltrating lobular carcinoma.

One of the many fascinating aspects of LISC is the mammographic findings. The presence of microcalcification in otherwise normal lobules adjacent to LISC was described by Hutter et al (1969). This could be the result of 'Pagetoid spread' in ducts, resulting in the damming up of secretions in adjacent normal lobules with consequent calcospherite formation. In view of our findings, another factor may well be qualitative and quantitative changes in mucins in the neoplastic lobules. Mucin (and possibly fragmented or whole tumour cells) may seep into neighbouring lobules served by the same ductal system, the secretion and/or fragments serving as a nidus for microcalcification. Thus, mechanical factors, alterations in mucin content, and doubtless other unrecognized factors may all play a rôle in the curious 'neighbourhood calcification' of LISC.

Our findings cast no light on why this mucinsecreting carcinoma has such a long in-situ phase in many patients. Still less do they solve the dilemma of the group of patients who do not develop an infiltrating carcinoma even after an interval of 23 years (McDivitt et al, 1967).

We wish to thank Professor K. Weinbren for his valuable advice and Miss Yvonne Maddison for expert technical assistance. We are indebted to Miss Debbie Hawks for typing the manuscript.

\footnotetext{
References

Carter, D., Yardley, J. H., and Shelley, W. M. (1969). Lobular carcinoma of the breast. An ultrastructural comparison with certain duct carcinomas and benign lesions. John Hopk. med. J., 125, 25-43.

Fechner, R. E. (1971). Ductal carcinoma involving the lobule of the breast. A source of confusion with lobular carcinoma in situ. Cancer (Philad.), 28, 274-281.

Foote, F. W., Jr. and Stewart, F. W. (1941). Lobular carcinoma in situ. Amer. J. Path., 17, 491-496.
} 
Gad, A. (1969). A histochemical study of human alimentary tract mucosubstances in health and disease. I. Normal and tumours. Brit. J. Cancer, 23, 52-63.

Hamperl, H. (1972). Zur Kenntnis des sog. Carcinoma lobuläre in situ der Mamma. Z. Krebsforsch., 77, 231-246.

Hutter, R. V. P., Snyder, R. E., Lucas, J. C., Foote, F. W., Jr., and Farrow, J. H. (1969). Clinical and pathologic correlation with mammographic findings in lobular carcinoma in situ. Cancer (Philad.), 23, 826-839.

McDivitt, R. W., Hutter, R. V. P., Foote, F. W., Jr., and Stewart, F. W. (1967). In situ lobular carcinoma. J. Amer. med. Ass., 201, 96-100.

McDivitt, R. W., Stewart, F. W., and Berg, J. W. (1968). Tumors of the Breast. Atlas of Tumour Pathology, 2nd series, Fasc. 2. Armed Forces Institute of Pathology, $\stackrel{\stackrel{2}{5}}{\overrightarrow{2}}$ Washington, D.C.

Newman, W. (1966). Lobular carcinoma of the female breast. Report of 73 cases. Ann. Surg., 164, 305-314.

Ozzello, L. (1971). Ultrastructure of the human mammary gland. In: Pathology Annual, vol. 6, edited by S. Sommers, p. 43. Butterworths, London.

Toker, C. (1974). Small cell dysplasia and in-situ carcinoma of the mammary ducts and lobules. J. Path., 114, 47-52.

Warner, N. E. (1969). Lobular carcinoma of the breast. Cancer (Philad.), 23, 840-846.

Wellings, S. R. and Roberts, P. (1963). Electron microscopy $\rightarrow$ of sclerosing adenosis and infiltrating duct carcinoma of the human mammary gland. $J$. nat. Cancer Inst., 30, 269-287. 